PROCEEDINGS OF THE

AMERICAN MATHEMATICAL SOCIETY

Volume 126, Number 4, April 1998, Pages 1205-1210

S 0002-9939(98)04246-4

\title{
A CHARACTERIZATION FOR SPACES OF SECTIONS
}

\author{
PALANIVEL MANOHARAN
}

(Communicated by Thomas Goodwillie)

\begin{abstract}
The space of smooth sections of a bundle over a compact smooth manifold $K$ can be equipped with a manifold structure, called an $A$-manifold, where $A$ represents the Fréchet algebra of real valued smooth functions on $K$. We prove that the $A$-manifold structure characterizes the spaces of sections of bundles over $K$ and its open subspaces. We also describe the $A^{(r)}$-maps between $A$-manifolds.
\end{abstract}

\section{INTRODUCTION}

The aim of this paper is to recognize among the infinite dimensional spaces those which are the spaces of smooth sections of bundles over a fixed compact connected manifold $K$. For this purpose, we use the concept of $A$-manifold structure, where $A$ is the Fréchet algebra of all real valued smooth functions on $K$. The idea of $A$-manifold structure in terms of local charts is explained in [3], and in terms of sheaves in [2]. Roughly, an $A$-manifold is a Hausdorff topological space which is locally modeled on finitely generated projective $A$-modules through $A$-maps, where an $A$-map is a map whose linear approximations are $A$-linear. One needs to be careful in proving results about $A$-manifolds and $A$-maps, because the partition of unity by $A$-maps does not exist on an $A$-manifold. It should be interesting to see, as an analogy to the finite dimensional case, whether every $A$-manifold can be embedded in $A^{\Lambda}$ for some index set $\Lambda$.

Let $\mathcal{M}$ be an $A$-manifold and $\Lambda=C_{A}^{\infty}(\mathcal{M})$ be the set of all $A$-maps from $\mathcal{M}$ to $A$. Unlike finite dimensional manifolds, $A$-manifolds as given in [2] and [3] do not have "bump" $A$-maps, and thus it still remains to be seen whether $\Lambda$ separates the points of $\mathcal{M}$, i.e., for every pair of distinct points $m_{1}, m_{2} \in \mathcal{M}$, whether there exists an $A$-map $F \in \Lambda$ such that $F\left(m_{1}\right) \neq F\left(m_{2}\right)$. If $\Lambda$ separates the points of $\mathcal{M}$, then $\mathcal{M}$ can considered as a subset of $A^{\Lambda}$. In this case, we can define an $A$-manifold (Definition 2.4) similarly to the definition of $n$-manifold given in [5]. Our main result gives a concrete realization of these $A$-manifolds.

A bundle over $K$ is a triple $M \stackrel{p}{\longrightarrow} K$, where $M$ is a finite dimensional manifold and $p$ is a surjective submersion. One can verify that the space of smooth sections $\Gamma M=\{s: K \rightarrow M \mid s \circ p=i d\}$ and its open subsets are equipped with $A$-manifold structure. Conversely, we prove that every $A$-manifold, as defined in

Received by the editors February 6, 1996 and, in revised form, September 17, 1996.

1991 Mathematics Subject Classification. Primary 58D15.

Key words and phrases. A-manifold, $A$-map, $A^{(r)}$-map.

The author was partially supported by NSF grant DMS-9401582.

The abstract was presented in the Joint Math Meeting, Orlando, January 1996. 
Definition 2.4, can be embedded as an open subset in $\Gamma M$ for some $M \stackrel{p}{\rightarrow} K$. Thus these $A$-manifolds characterize the spaces of sections of bundles over $K$ and its open subspaces.

In the last section, we briefly describe $A^{(r)}$-maps between two $A$-manifolds as a generalization of differential operators of order $r$.

\section{A-manifold structure}

Let us recall that there is a one-to-one correspondence between the category of smooth vector bundles over $K$ with bundle morphisms as maps and the category of finitely generated projective $A$-modules with module morphisms as maps [6]. More explicitly, for every smooth vector bundle $E \rightarrow K$, the corresponding finitely generated projective $A$-module is the space $\Gamma E$ of all smooth sections of $E \rightarrow K$. Hence every finitely generated projective $A$-module can be naturally equipped with a Fréchet space structure. An $A$-map between two finitely generated projective $A$-modules is a smooth map whose derivative at each point is $A$-linear.

It is shown in [3] that every fiber-preserving (not necessarily linear) map between two vector bundles induces an $A$-map between the corresponding spaces of sections, and, conversely, every $A$-map between two finitely generated projective $A$-modules is induced by a fiber-preserving map between the corresponding vector bundles over $K$.

We need the following discussion before giving the definition of $A$-manifold. Let $E \stackrel{p}{\longrightarrow} K$ be a smooth vector bundle. Consider the evaluation map $e v: \Gamma E \times K \longrightarrow$ $E$ defined by $e v(s, x)=s(x)$, which is a surjective smooth map.

Lemma 2.1. The evaluation map ev has a local right inverse at every point $e \in E$.

Proof. We prove this lemma by using the Nash-Moser-Hamilton inverse function theorem [1]. Notice that $e v: \Gamma E \times K \longrightarrow E$ is a smooth tame map. Let $x=p(e)$ and $W$ be a neighborhood of $x$ which is diffeomorphic to an open subset of $R^{k}$ such that $p^{-1}(W)$ is trivial. Locally on $W$, every section $s$ can be considered as a bounded smooth map with values in $R^{n}$. Consider ev $: \mathcal{U} \times W \longrightarrow R^{n}$, where $\mathcal{U}$ is an open subset of $C_{B}^{\infty}\left(W, R^{n}\right)$, the space of bounded smooth $R^{n}$-valued maps on $W$. Then, locally, the derivative

$$
D(e v):(\mathcal{U} \times W) \times\left(C_{B}^{\infty}\left(W, R^{n}\right) \times R^{k}\right) \longrightarrow R^{n}
$$

is given by

$$
D(e v)(s, x)(\alpha, v)=\alpha(x)+\frac{d s}{d x}(x)(v),
$$

where $(s, x) \in \mathcal{U} \times W$ and $(\alpha, v) \in C_{B}^{\infty}\left(W, R^{n}\right) \times R^{k}$. Since $R^{n}$ is finite dimensional, $D(e v)$ is a surjective tame map. By considering every element of $R^{n}$ as a constant map on $W$, one can assume that $R^{n} \subset C_{B}^{\infty}\left(W, R^{n}\right)$. Define

$$
(V e v):(\mathcal{U} \times W) \times T E \longrightarrow R^{n} \times R^{k} \subset C_{B}^{\infty}\left(W, R^{n}\right) \times R^{k}
$$

by

$$
(V e v)(s, x)(u)=\left(u_{v}, d p\left(u_{s}\right)\right),
$$

where $u_{v}$ is the vertical component of $u$ and $u_{s}$ is the $\frac{d s}{d x}$ component of $u \in T_{s(x)} E$.

Since $\operatorname{Im}(V e v)$ is finite dimensional, $(V e v)$ is tame. Thus $(V e v)$ is a smooth tame family of right inverses for $D(e v)$. Then Theorem 1.1.3 on p.172 of [1] implies that $e v$ is locally surjective and has a local right inverse at every point of $E$. 
Corollary 2.2. ev is an open map.

Proposition 2.3. Let $\mathcal{U}$ be a convex open subset of $\Gamma E$ and ev $(\mathcal{U} \times K)=U$, which is open in $E$ by the above corollary. For any given $A$-map $F: \mathcal{U} \rightarrow A$, there exists a unique smooth map $f: U \rightarrow R$ such that $F(s)=f \circ s$ for every $s \in \mathcal{U}$.

Proof. For any $e \in U$ and $x=p(e)$, choose a section $s \in \mathcal{U}$ such that $s(x)=e$. Define $f(e)=F(s)(x)$. By a similar proof as in Lemma 2.4 of [3], one can verify that $f$ is well-defined. We need to verify that $f$ is smooth. Let $\mu: O \rightarrow \mathcal{U} \times K$ be a local smooth right inverse of $e v$ at a neighborhood $O$ of $e$, and let $\pi_{1}: \mathcal{U} \times K \rightarrow \mathcal{U}$ and $\pi_{2}: \mathcal{U} \times K \rightarrow K$ be the projection maps. Then

$$
F\left(\pi_{1} \mu e\right)\left(\pi_{2} \mu e\right)=f\left(\pi_{1} \mu e\left(\pi_{2} \mu e\right)\right)=f(e v \circ \mu(e))=f(e) .
$$

Hence $f$ is smooth.

We now we give the definition of $A$-manifolds.

Definition 2.4. For any index set $\Lambda$, let $A^{\Lambda}$ be equipped with the product topology. A subset $\mathcal{M} \subset A^{\Lambda}$ is an $A$-manifold if for each $s \in \mathcal{M}$ there exists a smooth map $H: \mathcal{U} \rightarrow A^{\Lambda}$, defined on an open convex subset $\mathcal{U}$ of $\Gamma E$, where $E \stackrel{p}{\longrightarrow} K$ is a smooth vector bundle of rank $n$, such that

1. $H$ is an $A$-map, or in other words, the composition $\mathcal{U} \stackrel{H}{\rightarrow} A^{\Lambda} \stackrel{p r_{\lambda}}{\rightarrow} A$ is an $A$-map for each projection $p r_{\lambda}$ for all $\lambda \in \Lambda$.

2. $H$ maps $\mathcal{U}$ homeomorphically onto a neighborhood $\mathcal{V}$ of $s \in \mathcal{M}$.

3. For each $t \in \mathcal{U}, D H(t)$ is injective.

4. By the previous proposition, $H$ is induced by a unique map $h: U \rightarrow R^{\Lambda}$. For each $x \in K, h_{x}$ maps $U_{x}=p^{-1}(x) \cap U$ homeomorphically onto a neighborhood $V_{x}$ of $s(x) \in M_{x}$, where $M_{x}=e v(\mathcal{M} \times x)$.

As usual, one may call the pair $(\mathcal{U}, H)$ a chart for $\mathcal{M}$, and a collection of charts which cover $\mathcal{M}$ an atlas.

Remark. If $K=\{p t\}$, then $A=R$, and the above definition is simply the definition of $n$-dimensional manifolds as given in [5]. It may be interesting to see whether condition 4 in the above definition is independent of the first three conditions.

Example 2.5. If $M \rightarrow K$ is a bundle, then the space of all sections $\Gamma M$ is an $A$-manifold.

Proof. Let $\Lambda=C^{\infty}(M)$. $\Gamma M$ can be considered as a subset of $A^{\Lambda}$ by defining $i: \Gamma M \longrightarrow A^{\Lambda}$ by $i(\gamma)_{\lambda}=\lambda \circ \gamma$ for each $\gamma \in \Gamma M$ and $\lambda \in \Lambda$. $\Gamma M$ is locally modeled near $\gamma \in \Gamma M$ by $\Gamma\left(\gamma^{*} T_{v} M\right)$, the sections of the pull-back of the vertical tangent bundle. Indeed, one can find an explicit construction of a homeomorphism $\Phi: \mathcal{U} \rightarrow$ $\Phi(\mathcal{U}) \subset \Gamma M$ in Proposition 3.5 of [3], where $\mathcal{U}$ is a convex open neighborhood of the zero section of $\gamma^{*} T_{v} M \rightarrow K$. For each such $(\mathcal{U}, \Phi)$, simply define $H: \mathcal{U} \rightarrow A^{\Lambda}$ by $H=i \circ \Phi$ and see that $H$ satisfies the conditions of the above definition.

Example 2.6. As an open subset of an $A$-manifold, every open subset of $\Gamma M$ is itself an $A$-manifold. 


\section{Embedding of $A$-MANifolds}

In this section we show that the space of sections of the bundles and its open subsets are the only $A$-manifolds as defined in 2.4.

Let $\mathcal{M}$ be an $A$-manifold as defined in 2.4. For every chart $(\mathcal{U}, H)$ of $\mathcal{M}$, Proposition 2.3 implies that $H$ induces a unique smooth map $h: U \rightarrow R^{\Lambda}$ and thus a unique map $\bar{h}: U \rightarrow R^{\Lambda} \times K$ defined by $\bar{h}(e)=(h(e), p(e))$, where $p: U \rightarrow K$ is the restriction of the bundle projection $E \stackrel{p}{\longrightarrow} K$.

Proposition 3.1. The map $\bar{h}: U \rightarrow R^{\Lambda} \times K$ is such that $d \bar{h}(e)$ is injective for every $e \in U$ and $\bar{h}$ maps $U$ homeomorphically onto $\bar{h}(U)$.

Proof. Let $e \in U$ and $v \in T_{e} U$, where $T_{e} U$ is the tangent space of $U$ at $e$. Suppose that $d \bar{h}(e)(v)=(d h(e)(v), d p(e)(v))=0 . d p(e)(v)=0$ implies that $v$ is a vertical tangent vector in $T_{e} U$. One can choose $s \in \mathcal{U}$ and $t \in \Gamma E$ such that $s(x)=e$ and $t(x)=v \cdot d h(e)(v)=0$ implies that $D H(s)(t)_{x}=0$. Let us say that $\left\{s_{i}\right\}$ is a local base near $x$, and $t=\sum_{i} \alpha_{i} s_{i}$ in this local coordinate system. Then $0=D H(s)(t)_{x}=D H(s)\left(\sum_{i} \alpha_{i} s_{i}\right)_{x}=\sum_{i} \alpha_{i}(x) D H(s)\left(s_{i}\right)_{x}$, since $D H(s)$ is $A$ linear. But the injectivity of $D H(s)$ implies that $\left\{D H(s)\left(s_{i}\right)_{x}\right\}_{i=1}^{n}$ are linearly independent. Therefore $\alpha_{i}(x)=0$ for all $i$, which shows that $v=t(x)=0$. Thus $d \bar{h}(e)$ is injective for each $e \in U$.

Next we verify that $\bar{h}$ is injective. Suppose that $e_{1}, e_{2} \in U$ and $\bar{h}\left(e_{1}\right)=\bar{h}\left(e_{2}\right)$. $p\left(e_{1}\right)=p\left(e_{2}\right)$ implies that $e_{1}$ and $e_{2}$ are in the same fiber, say in $U_{x}$. Since $h\left(e_{1}\right)=h\left(e_{2}\right)$ and $h_{x}$ is injective on $U_{x}$, we have $e_{1}=e_{2}$.

Since $d \bar{h}(e)$ is injective for each $e \in U, \bar{h}$ is one-to-one, and $h_{x}$ maps $U_{x}$ homeomorphically onto its image, it follows that $\bar{h}$ maps $U$ homeomorphically onto $\bar{h}(U)$.

Theorem 3.2. Every A-manifold $\mathcal{M}$ can be embedded as an open subset in $\Gamma(M \rightarrow K)$ for some bundle $M \rightarrow K$.

Proof. Let $\mathcal{M} \subset A^{\Lambda}$ be an $A$-manifold. We will construct an $(n+k)$-dimensional manifold $M \subset R^{\Lambda} \times K$ such that $M$ is a bundle over $K$, and show that $\mathcal{M}$ is embedded as an open subset in $\Gamma M$.

Each chart $(\mathcal{U}, H)$ of $\mathcal{M}$ induces $\bar{h}: U \rightarrow R^{\Lambda} \times K$ such that $d \bar{h}(e)$ is injective for each $e \in U$ and maps $U$ homeomorphically onto $\bar{h}(U) \subset R^{\Lambda} \times K$ by the previous proposition. Let $\left\{\left(\mathcal{U}_{\alpha}, H_{\alpha}\right)\right\}_{\alpha}$ be an atlas for $\mathcal{M}$. Let $M=\bigcup_{\alpha} \bar{h}_{\alpha}\left(U_{\alpha}\right)$. One can see that $M$ is a manifold of dimension $n+k$ with atlas $\left\{\left(U_{\alpha}, \bar{h}_{\alpha}\right\}_{\alpha}\right.$. Each $\bar{h}_{\alpha}$ is fiber-preserving, which implies that there exists a projection $p: M \rightarrow K$ which is a surjective submersion.

Since each $\mathcal{U}_{\alpha}$ is an open subset of $\Gamma U_{\alpha}$ and $\bar{h}_{\alpha}\left(U_{\alpha}\right)$ is open in $M$, there exists $i_{\alpha}: H_{\alpha}\left(\mathcal{U}_{\alpha}\right) \hookrightarrow \Gamma M$, embedded as an open subset. Each $i_{\alpha}$ and $i_{\beta}$ agree on $H_{\alpha}\left(\mathcal{U}_{\alpha}\right) \bigcap H_{\beta}\left(\mathcal{U}_{\beta}\right)$, which implies that there exists a unique map $i: \mathcal{M} \hookrightarrow \Gamma M$ such that $i(\mathcal{M})=\bigcup_{\alpha} i_{\alpha}\left(H_{\alpha}\left(\mathcal{U}_{\alpha}\right)\right)$ is open in $\Gamma M$.

Remark. If $K=\{p t\}$, the above theorem simply states that every finite dimensional manifold $M$ can be considered as the space of sections of the bundle $M \rightarrow\{p t\}$.

Let $M_{1} \rightarrow K$ and $M_{2} \rightarrow K$ be any two bundles. It is shown in [3] that every fiber-preserving map $f: M_{1} \rightarrow M_{2}$ induces the $A$-map $\Gamma f: \Gamma M_{1} \rightarrow \Gamma M_{2}$, and, conversely, every $A$-map $\Phi: \Gamma M_{1} \rightarrow \Gamma M_{2}$ is of the form $\Gamma f$ for some $f$. 
Proposition 3.3. Let $\mathcal{M}$ be embedded as an open subset in $\Gamma M$ as in the above theorem. Then every A-map $F: \mathcal{M} \rightarrow A$ can be uniquely extended to an A-map $\Gamma f: \Gamma M \rightarrow A$.

Proof. Let $\left\{\left(\mathcal{U}_{\alpha}, H_{\alpha}\right)\right\}_{\alpha}$ be an atlas for $\mathcal{M}$ and let $F: \mathcal{M} \rightarrow A$ be an $A$-map. For each $\alpha, F \circ H_{\alpha}: \mathcal{U}_{\alpha} \rightarrow A$ is an $A$-map. This implies that there exists a unique $f_{\alpha}: U_{\alpha} \rightarrow R$ such that $\Gamma f_{\alpha}=F \circ H_{\alpha}$ by Proposition 2.3, and hence there exists a unique $\tilde{f}_{\alpha}: h_{\alpha}\left(U_{\alpha}\right) \rightarrow R$. By uniqueness, $\tilde{f}_{\alpha}$ and $\tilde{f}_{\beta}$ agree on $h_{\alpha}\left(U_{\alpha}\right) \cap h_{\beta}\left(U_{\beta}\right)$ and hence induce a unique map $f: M \rightarrow R$, which yields $\Gamma f: \Gamma M \rightarrow A$.

\section{Differential operators}

In the language of category theory, we characterized $A$-manifolds in the last section which are the objects of our category. The natural choice for the maps of our category are $A$-maps. A smooth map $\Phi: \mathcal{M} \rightarrow \mathcal{N}$ between two $A$-manifolds $\mathcal{M}$ and $\mathcal{N}$ is an $A$-map if $D_{s} \Phi: T_{s} \mathcal{M} \rightarrow T_{\Phi(s)} \mathcal{N}$, the derivative of $\Phi$ at each $s \in \mathcal{M}$, is $A$-linear, where $T_{s} \mathcal{M}$ is the tangent space of $\mathcal{M}$ at $s$. Unfortunately, the collection of $A$-maps is too 'small'. For example, if $K=S^{1}$ then the first order differential operator $\Phi: C^{\infty}\left(S^{1}\right) \rightarrow C^{\infty}\left(S^{1}\right)$ defined by $\Phi(f)=f^{\prime}$ is not an $A$-map. We can 'enlarge' the class of maps in our category by including $A^{(r)}$-maps, which generalize A-maps.

Definition 4.1. A smooth map $\Phi: \mathcal{M} \rightarrow \mathcal{N}$ is called an $A^{(r)}$-map if

$$
\left(D_{s} \Phi\right)\left(\mathbf{m}^{r+1} T_{s} \mathcal{M}\right) \subset \mathbf{m} T_{\Phi(s)} \mathcal{N}
$$

for every maximal ideal $\mathbf{m}$ of $A$ and for every $s \in \mathcal{M}$.

Let $E_{1}, E_{2} \rightarrow K$ be any two smooth vector bundles and $j^{r} E_{1} \rightarrow K$ be the $r$-jet bundle of $E_{1} \rightarrow K$. A non-linear differential operator of order $r$ between $\Gamma E_{1}$ and $\Gamma E_{2}$ is a smooth map $\Phi: \Gamma E_{1} \rightarrow \Gamma E_{2}$ defined by $\Phi(s)=\phi \circ j^{r}(s)$ for some fiber-preserving map $\phi: j^{r} E_{1} \rightarrow E_{2}$. It is verified in [4] that a smooth map $\Phi: \Gamma E_{1} \rightarrow \Gamma E_{2}$ is a non-linear differential operator of order $r$ if and only it is an $A^{(r)}$-map.

Of course, when $\mathcal{M}=\Gamma M$ and $\mathcal{N}=\Gamma N$, the above definition includes the standard non-linear differential operators.

Example 4.2. Let $M, N \rightarrow K$ be any two bundles and let $j^{r} M \rightarrow K$ be the $r$ jet bundle of $M \rightarrow K$. If $\phi: j^{r} M \rightarrow N$ is a fiber-preserving smooth map, then $\Phi: \Gamma M \rightarrow \Gamma N$, defined as $\Phi(s)=\phi \circ j^{r}(s)$, is an $A^{(r)}$-map.

Proof. Let $s \in \Gamma M$. Choose a chart $\Gamma U \subset \Gamma E_{1}$ at $s$ and a chart $\Gamma V \subset \Gamma E_{2}$ at $\Phi(s)$. We wish to show that $\left(D_{s} \Phi\right)\left(\mathbf{m}^{r+1} \Gamma E_{1}\right) \subset \mathbf{m} \Gamma E_{2}$.

Now $D_{s} \Phi=\left(D_{j^{r} s} \Gamma \phi\right) \circ D_{s} j^{r}$. Since $\Gamma \phi$ is an $A$-map, it is enough to show that $\left(D_{s} j^{r}\right)\left(\mathbf{m}^{r+1} \Gamma E_{1}\right) \subset \mathbf{m}\left(j^{r} \Gamma E_{1}\right)$, which immediately follows from Lemma 2.4 of [4], because $\left(D_{s} j^{r}\right)(h)=j^{r} h$.

\section{ACKNOWLEDGMENTS}

I wish to thank Professor D. Burghelea for his discussions with me regarding this paper, and the referee for his suggestion on the original version of this paper. 


\section{REFERENCES}

1. Hamilton, R.S.: The inverse function theorem of Nash and Moser. Bull. Am. Math. Soc. 7 no. 1, 65-222 (1982). MR 83j:58014

2. Kobayashi, S.: Manifolds over function algebras and mapping spaces. Tôhoku Math. J. 41, 263-282 (1989). MR 90g:58014

3. Manoharan, P.: A non-linear version of Swan's theorem. Math. Z. 209, 467-479 (1992). MR 93d:55019

4. Manoharan, P.: Generalized Swan's theorem and its application. Proc. Amer. Math. Soc. 123, no. 10, 3219-3223 (1995). MR 95m:58020

5. Milnor, J.W. and Stasheff, J.D.: Characteristic classes, Princeton University Press, 1974. MR $\mathbf{5 5 : 1 3 4 2 8}$

6. Swan, R.G.: Vector bundles and projective modules. Trans. Amer. Math. Soc. 105, 264-277 (1962). MR 26:785

Department of Mathematics, Kent State University, East Liverpool, Ohio 43920

E-mail address: manohara@mcs.kent.edu

Current address: College of Arts and Sciences, Florida Gulf Coast University, Fort Myers, Florida 33965 